\section{ANÁLISE DA VIABILIDADE DE APROVEITAMENTO DE ÁGUA DA CHUVA PARA FINS NÃO POTÁVEIS EM EDIFICAÇÃO DA UNIVERSIDADE FEDERAL DO OESTE DO PARÁ - SANTARÉM}

\author{
ANALYSIS OF THE FEASIBILITY OF USE OF RAINWATER \\ FOR NON-POTABLE PURPOSES IN BUILDING OF THE \\ FEDERAL UNIVERSITY OF OESTE DO PARÁ - SANTARÉM
}

\footnotetext{
Isaías Conceição Gonçalves Universidade Federal do Pará PPCS - Programa de PósGraduação em Processos Construtivos e Ambiental. E-mail: goncalvesisaias@hotmail.com

Claudio José Cavalcante Blanco

Universidade Federal do Pará FAESA - Faculdade de Engenharia Sanitária e Ambiental Recursos Hídricos. E-mail: blanco@ufpa.br
}

\section{RESUMO}

Este estudo avalia o potencial de aproveitamento de água de chuva em uma edificação da Universidade do Oeste do Pará (UFOPA). A metodologia seguiu a norma da ABNT/NBR- 15527:2019, com destaque para a seleção e o dimensionamento do reservatório do sistema via método da simulação, buscando a avaliação do tripé de sustentabilidade ambiental, viabilidade econômica e social. Após simulações para determinação do reservatório do sistema, determinouse um reservatório com volume de $274 \mathrm{~m}^{3}$. Esse valor atenderia a demanda integral de água para usos não potáveis da edificação. Entretanto, a dimensão desse reservatório impede sua implantação devido à falta de espaço às proximidades da edificação. Assim, optouse pela simulação do reservatório com um volume de $78 \mathrm{~m}^{3}$, obtendose um custo de $\mathrm{R} \$ 82.982,59$ para a implantação do sistema. Apesar do sistema de abastecimento da Universidade ser realizado por extração própria de poço profundo. Realizou-se a análise para se ter uma confrontação com valor de mercado da Companhia de Saneamento, que para o consumo estipulado totalizou mensalmente uma tarifa de $\mathrm{R} \$ 2.159,01$. Os resultados obtidos indicaram uma possibilidade de suplementação ou redução do consumo de água potável nos banheiros da edificação escolhida por um período de 9 meses e com uma necessidade de suplementar o sistema projetado nos 3 meses de estiagem da região.

Palavras-chave: Aproveitamento de água da chuva, Regulamentação, Viabilidade, Sustentabilidade.

\begin{abstract}
This study evaluates the potential for using rainwater in a building at the University of Oeste do Pará (UFOPA). The methodology followed the ABNT/NBR-15527/2019 standard, with emphasis on the selection and sizing of the system's reservoir via the simulation method, seeking to assess the tripod of environmental sustainability, economic and social feasibility. After simulations to determine the system's reservoir, a reservoir with a volume of $274 \mathrm{~m}^{3}$ was determined. This amount would meet the entire water demand for non-potable uses of the building. However, the size of this reservoir prevents its implementation due to the lack of space in the vicinity of the building. Thus, we opted for the simulation of the reservoir with a volume of 78
\end{abstract}


$\mathrm{m}^{3}$, obtaining a cost of $\mathrm{R} \$ 82,982.59$ for the implementation of the system. Despite the University's supply system being carried out by its own deep well extraction. The analysis was carried out to have a comparison with the Sanitation Company's market value, which for the stipulated consumption totaled a monthly fee of $R \$ 2,159.01$. The results obtained indicated a possibility of supplementing or reducing the consumption of drinking water in the bathrooms of the chosen building for a period of 9 months and with a need to supplement the projected system in the 3 months of drought in the region.

Keywords: Use of rainwater, Regulation, Feasibility, Sustainability 


\section{INTRODUÇÃO}

A impermeabilização devido à urbanização, que muitas vezes é desordenada, causa alagamentos, os quais são agravados pelo descarte inadequado de resíduos sólidos, que obstruem o sistema de drenagem, que geralmente, é insuficiente e deficiente. Lambertucci et al. (2016) comentam sobre o aproveitamento de água de chuva como uma das técnicas sustentáveis que pode contribuir na obtenção de resultados positivos no controle de águas pluviais e prevenção de enchentes com o retardo no tempo de concentração de águas pluviais na drenagem urbana. Magalhães et al. (2009) mostraram que os eventos de chuva são responsáveis pelo aumento de doenças de veiculação hídricas, entre elas a leptospirose, além de Zika, Dengue e Chincungunya com vetores que se desenvolvem onde exista acúmulo de água.

Assim, a gestão adequada dos recursos hídricos via procedimentos como reuso e o aproveitamento de água da chuva é importante para minimizar os desperdícios e atender várias demandas que não exigem potabilidade. Em congresso mundial organizado em 2018 pela International Water Association (IWA, 2018), o tema água foi discutido com assuntos conexos que provocam as crises hídricas, alteram o volume disponível e aceleram a escassez. Entre esses assuntos foram destacados as mudanças climáticas, crescimento populacional, crise global de saneamento e urbanização.

No Brasil, na cidade do Samba, no município do Rio de Janeiro-RJ, existe um sistema de captação de água da chuva com $20.000 \mathrm{~m}^{2}$ e reservatórios de até $300 \mathrm{~m}^{3}$, que abastecem os vasos sanitários e torneiras para lavagens de galpões. Os estádios da copa do mundo de 2014 foram projetados e executados com sistema de aproveitamento de água da chuva, por exemplo, Estádio Mineirão, em Belo Horizonte-MG, com um sistema de aproveitamento de água da chuva com reservatório de $6.000 \mathrm{~m}^{3}$. Essa água é utilizada para abastecer os vasos sanitários, na limpeza externa e irrigação do gramado. Também a Arena Fonte Nova, na cidade de Salvador-BA, foi construído com um sistema de captação de água da chuva e armazenamento para utilização na irrigação do gramado e sanitários (PEREIRA et al., 2019).

No contexto do presente trabalho, recentemente, Cardoso et al. (2020) analisaram a viabilidade econômica e o dimensionamento de um sistema de captação de água da chuva para fins não potáveis de dois prédios da Universidade Federal do Pará (UFPA). Os autores concluíram que a implementação do sistema é viável do ponto de vista 
econômico e ambiental quando aplicado em prédios públicos que consomem água da concessionária, uma vez que a substituição da água potável pela água da chuva em usos não potáveis, como em aparelhos sanitários e limpeza dos prédios, acarretaria consideráveis economias nos gastos públicos. Assim, o estudo pretende analisar a viabilidade de um sistema de aproveitamento de água da chuva na Universidade Federal do Oeste do Pará (UFOPA), buscando atender os usos não potáveis de água de um bloco de sala de aulas.

\section{MATERIAL E MÉTODOS}

\section{Área de Estudo}

A Universidade Federal do Oeste do Pará (UFOPA) criada no ano de 2009 é proveniente da fusão do patrimônio do Campus de Interiorização da UFPA (Universidade Federal do Pará) e UFRA (Universidade Federal Rural da Amazônia). A edificação escolhida para este estudo denominada Salas Especiais "Laranjão" encontrase no Campus Tapajós, sendo a segunda edificação executada no início das atividades da UFOPA, estando localizada no seu campus principal na cidade de Santarém (Figura 1).

Figura 1 - Imagem Satélite de região da cidade de Santarém e em destaque a UFOPA

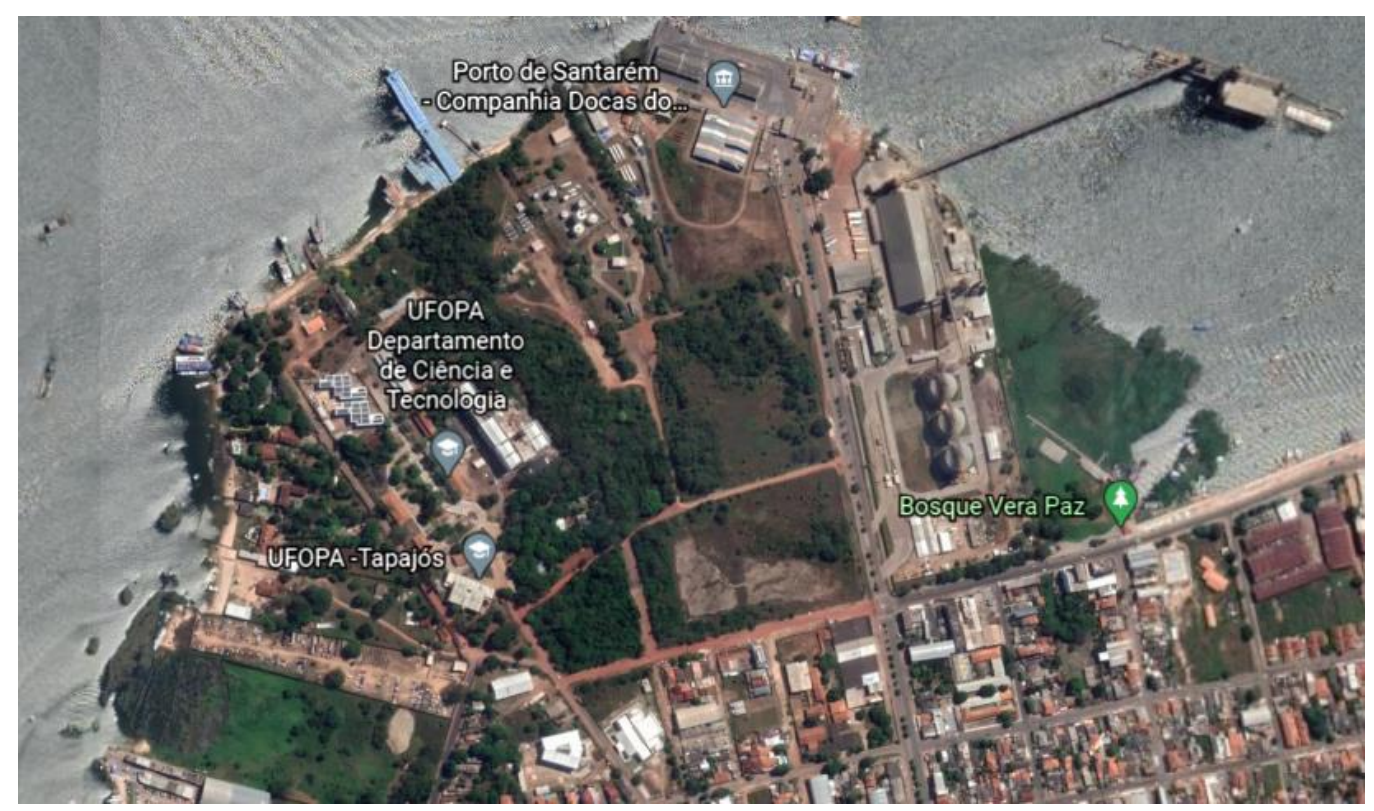

Fonte: Google Earth (2020) 
O projeto do sistema de captação de água da chuva do prédio Laranjão (Figura 2) foi desenhado para atender aos vasos sanitários dos sanitários existentes nos banheiros masculino e feminino do prédio, reduzindo a utilização de água potável para esta finalidade ou seu atendimento integral. A população analisada foi a de alunos que frequentam as aulas, considerando-se a capacidade máxima das salas e a utilização dos vasos sanitários pelos alunos ao longo do dia, totalizando-se mensalmente o volume necessário de atendimento. Nesse caso, edificação possui uma área de captação de água da chuva em telha metálica de $1607 \mathrm{~m}^{2}$.

Figura 2 - Prédio “Laranjão” no Campus Tapajós da UFOPA em Santarém-PA

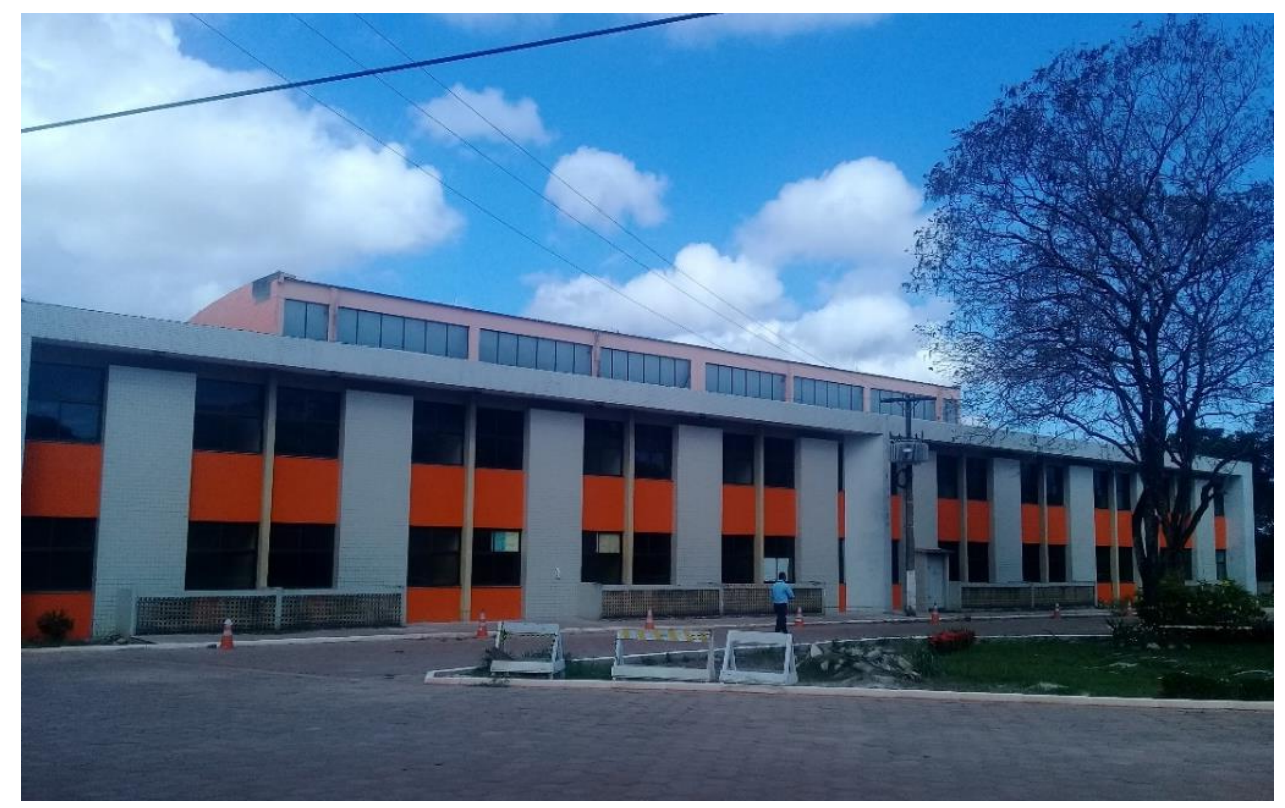

Fonte: Autor (2020)

\section{Métodos para dimensionamento de reservatórios}

Os métodos para dimensionamento de reservatórios existentes de acordo com a NBR (ABNT, 2019) são os seguintes, de forma resumida, método de Rippl, Simulação, Azevedo Neto, Prático Alemão, Prático Inglês, Australiano. Neste trabalho, foi utilizado o Método de Simulação. Segundo Rupp et al. (2011), o método de simulação baseia-se no percentual de atendimento de consumo em função de um volume de reservatório definido previamente. Neste método a evaporação da água é desprezada. Assim, para um determinado mês, aplica-se a equação da continuidade (Equação 1) a um reservatório finito. 


$$
\begin{aligned}
& S(t)=Q(t)+S(t-1)-D(t) \\
& \text { Sendo } 0<S(t)<V
\end{aligned}
$$

Onde $\mathrm{Q}(\mathrm{t})$ é o volume de chuva no tempo $\mathrm{t}$; $\mathrm{S}(\mathrm{t})$ é o volume de água do reservatório; $\mathrm{S}(\mathrm{t}-1)$ é o volume de água do reservatório no tempo $(\mathrm{t}-1) ; \mathrm{D}(\mathrm{t})$ é o volume do reservatório.

De acordo com ABNT (2019), o volume de água de chuva aproveitável depende do coeficiente de escoamento superficial da cobertura, bem como da eficiência do sistema de descarte do escoamento inicial, sendo calculado pela Equação 2.

$$
\mathrm{Q}(\mathrm{t})=\mathrm{C} \cdot \eta \cdot \mathrm{P}(\mathrm{t}) \cdot \mathrm{A}
$$

Onde C é o coeficiente de escoamento superficial (-); P(t) é lâmina de chuva no tempo t; e A é área de Captação $\left(\mathrm{m}^{2}\right)$. e $\eta$ é o fator de captação (-). O fator de captação é a eficiência do sistema, levando em conta o dispositivo de descarte de sólidos e desvio de escoamento inicial, caso este último seja utilizado. Um valor prático quando não se têm dados é adotar: C. $\eta=0,80$ (MUNDT et al., 2013). Esse valor foi adotado no presente trabalho.

Na análise abordada, o tempo "t" foi medido em meses. Além disso, para este método, duas hipóteses foram consideradas, o reservatório está cheio no início da contagem do tempo “t” e os dados históricos são representativos para as condições futuras.

\section{Dados Pluviométricos}

As séries históricas de chuva da cidade de Santarém entre os anos de 1968 a 2015 oriundos da estação pluviométrica Código: 254000, disponível no Hidroweb do Sistema Nacional de Informações sobre Recursos Hídricos da ANA (http://www.snirh.gov.br/hidroweb/serieshistoricas). Na Figura 3 é mostrada a distribuição mensal de chuvas de Santarém. 
Figura 3 - Pluviograma mensal médio da cidade de Santarém/PA

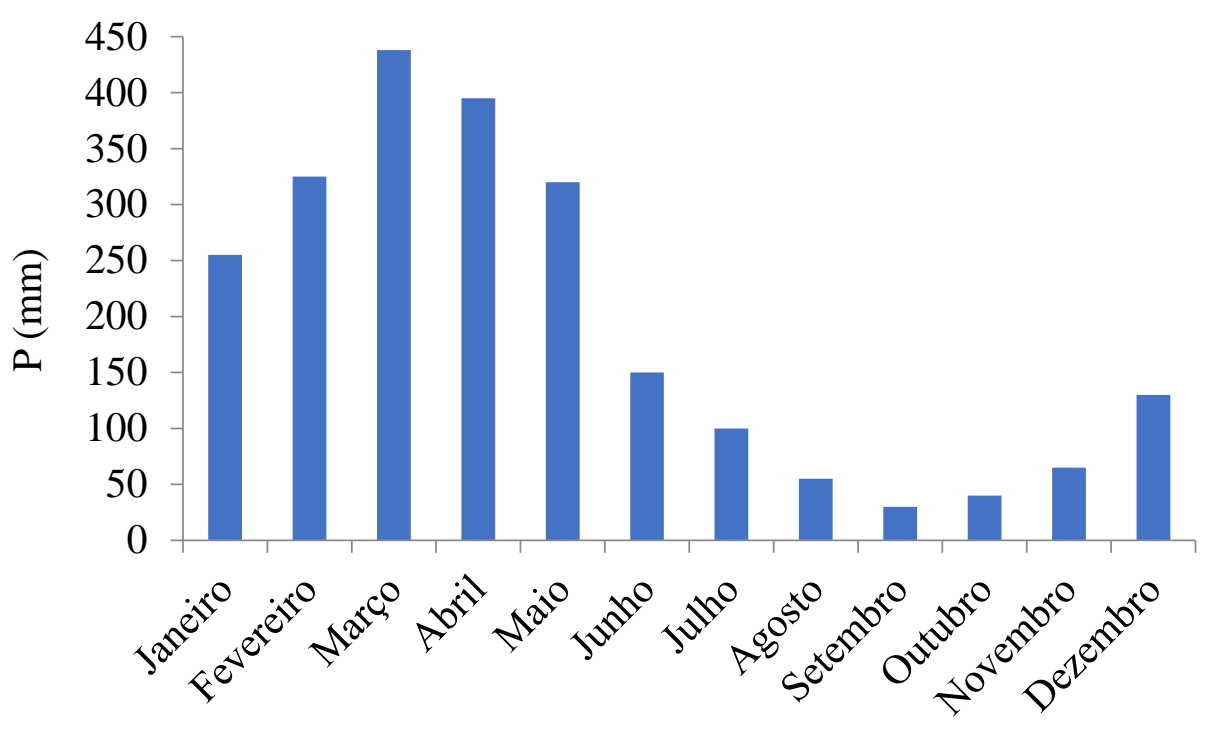

Fonte: Autor (2020)

Com base na Figura 3, observa-se em Santarém a existência de duas estações uma estação chuvosa que vai de dezembro a maio e uma estação seca, que vai de junho a novembro. Essa sazonalidade reflete-se no funcionamento dos sistemas de aproveitamento de água da chuva da região. Assim, os sistemas funcionam com sobra de água no primeiro semestre e falta no segundo semestre (Da CRUZ e BLANCO, 2017; CARDOSO et al., 2020).

\section{Demanda de água para descargas sanitárias}

A Tabela 1 apresenta as variáveis que possibilitam a quantificação do volume de água necessário para atender a demanda mensal das descargas sanitárias do prédio de salas de aula analisado.

Tabela 1 - Demanda da edificação para uso de água da chuva em caixas de descargas

\begin{tabular}{ccccc}
\hline $\begin{array}{c}\mathbf{N}^{\mathbf{0}} \text { de } \\
\text { usuários }\end{array}$ & $\begin{array}{c}\mathbf{N}^{\circ} \text { de } \\
\text { acionamentos }\end{array}$ & $\begin{array}{c}\text { Volume } \\
\left(\mathbf{m}^{\mathbf{3}}\right) \text { /Acionamento }\end{array}$ & $\begin{array}{c}\mathbf{N}^{\circ} \text { de dias } \\
\text { utilizados }\end{array}$ & $\begin{array}{c}\text { Volume total } \\
\left(\mathbf{m}^{\mathbf{3}} / \mathbf{m e ̂ s}\right)\end{array}$ \\
\hline 500 & 2 & 0,006 & 22 & 132 \\
\hline \multicolumn{5}{c}{ Fonte: Autor (2020) }
\end{tabular}

Nesse caso, foram computados os dias uteis efetivamente de alta concentração de pessoas na edificação. Observando-se também que não foram reduzidas as demandas no 
Revista Tecnologia e Ambiente, v. 28, 2022, Criciúma, Santa Catarina/SC - ISSN

Eletrônico 2358-9426 e ISSN Impresso 1413-8131

período de férias para se ter uma avaliação mais desfavorável da necessidade de água. Quanto ao número de acionamento foram considerados 2 acionamentos por pessoas devido à característica de rotatividade dos usuários e a existência de sanitários em outras edificações do campus.

\section{Tabela de tarifas da companhia de saneamento}

Na Figura 4 são apresentadas as tarifas por faixa de consumo da Companhia de Saneamento do Pará (COSANPA, 2020), válida a partir de 14/12/2019. Os dados dessa tabela foram usados para determinação do payback do investimento.

Figura 4 - Valores de Tarifas da Companhia de Saneamento do Pará

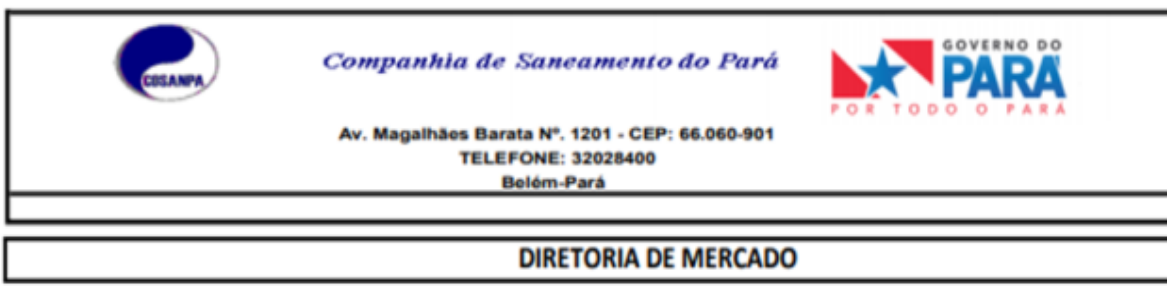

\begin{tabular}{|c|c|c|c|}
\hline \multicolumn{4}{|c|}{ TABELA TARIFARIA a partir de 14/dezembro/2019 - COSANPA } \\
\hline CATEGORIA & FADXA de CONSUMO & VALOR da ÁGUA & VALOR dO ESGOTO \\
\hline \multirow{6}{*}{ RESIDENCIAL } & $0-10$ & 2,94 & 1,76 \\
\hline & $11-20$ & 4,20 & 2,52 \\
\hline & $21 \cdot 30$ & 5,63 & 3,38 \\
\hline & $31 \cdot 40$ & 6,34 & 3,80 \\
\hline & 41.50 & 8,79 & 5,27 \\
\hline & $>50$ & 11,42 & 6,85 \\
\hline \multirow{2}{*}{ COMERCIAL } & $0-10$ & 8,79 & 5,27 \\
\hline & $>10$ & 10,97 & 6,58 \\
\hline \multirow{2}{*}{ INDUSTRIAL } & $0-10$ & 10,97 & 6,58 \\
\hline & $>10$ & 14,04 & 8,43 \\
\hline \multirow{2}{*}{ PÚBLICA } & $0-10$ & 8,79 & 5,27 \\
\hline & $>10$ & 10,97 & 6,58 \\
\hline
\end{tabular}

\begin{tabular}{|c|c|c|c|c|c|c|c|c|}
\hline \multicolumn{3}{|c|}{ Tarifas } & \multicolumn{6}{|c|}{ Esgoto $=60 \%$ água } \\
\hline Categoria & Sub-categoria & Quantidade & & & Valc & esgoto & Águ & + Esgoto \\
\hline \multirow[t]{4}{*}{ Residencial } & R1 & $10 \mathrm{~m} 3$ & RS & 29,40 & RS & 17,64 & RS & 47,04 \\
\hline & R2 & $20 \mathrm{~m} 3$ & RS & 71,40 & RS & 42,84 & RS & 114,24 \\
\hline & R3 & $30 \mathrm{m3}$ & RS & 127,70 & RS & 76,62 & RS & 204,32 \\
\hline & R4 & $40 \mathrm{~m} 3$ & RS & 191,10 & RS & 114,66 & RS & 305,76 \\
\hline \multirow[t]{4}{*}{ Comercial } & $\mathrm{C1}$ & $10 \mathrm{~m} 3$ & RS & 87,90 & RS & 52,74 & RS & 140,64 \\
\hline & $\mathrm{C2}$ & $25 \mathrm{m3}$ & RS & 252,45 & RS & 151,47 & RS & 403,91 \\
\hline & $\mathrm{C3}$ & $50 \mathrm{m3}$ & RS & 526,70 & RS & 316,02 & RS & 842,72 \\
\hline & $\mathrm{C4}$ & $75 \mathrm{~m} 3$ & RS & 800,95 & RS & 480,57 & RS & $1.281,51$ \\
\hline \multirow[t]{4}{*}{ Industrial } & I1 & $10 \mathrm{~m}^{3}$ & RS & 109,70 & RS & 65,82 & RS & 175,52 \\
\hline & 12 & $25 \mathrm{m3}$ & RS & 320,30 & RS & 192,18 & RS & 512,48 \\
\hline & 13 & $50 \mathrm{m3}$ & RS & 671,30 & RS & 402,78 & RS & $1.074,08$ \\
\hline & 14 & $75 \mathrm{~m} 3$ & RS & $1.022,30$ & RS & 613,38 & RS & $1.635,68$ \\
\hline \multirow[t]{4}{*}{ Público } & P1 & $10 \mathrm{~m}^{3}$ & RS & 87,90 & RS & 52,74 & RS & 140,64 \\
\hline & $P 2$ & $25 \mathrm{m3}$ & RS & 252,45 & RS & 151,47 & RS & 403,91 \\
\hline & P3 & $50 \mathrm{m3}$ & RS & 526,70 & RS & 316,02 & RS & 842,72 \\
\hline & P4 & $75 \mathrm{~m} 3$ & RS & 800,95 & RS & 480,57 & RS & $1.281,51$ \\
\hline
\end{tabular}

Fonte: COSANPA (2020) 


\section{Critérios para análise de viabilidade do sistema}

Os critérios Confiança (Equações 3 e 4), Eficiência (Equação 5) e Payback (Equação 7) são ferramentas que permitem avaliar a viabilidade do sistema. A confiança é representada pelo percentual de segurança quanto à adequabilidade do dimensionamento no atendimento da demanda; a Eficiência do Sistema também é proporcional ao aproveitamento máximo de água da chuva disponível; e o payback representa o período de retorno do investimento financeiro na construção do sistema de aproveitamento de água da chuva.

$$
\begin{aligned}
& \mathrm{Rf}=(1-\mathrm{Fr}) \cdot 100 \\
& \mathrm{Fr}=\mathrm{Nr} / \mathrm{N}
\end{aligned}
$$

Onde Rf é a confiança do sistema (\%); Fr é a falha; Nr é o número de meses em que o sistema não atendeu a demanda; e $\mathrm{N}$ é o número de meses considerado, nesse caso 12 meses (1 ano);

A eficiência do sistema é dada pela Equação 5, considerando-se a relação entre o volume anual de chuva aproveitada pelo sistema e o volume de chuva anual.

$$
\mathrm{Ef}=(\mathrm{VCA} / \mathrm{VC}) .100
$$

Onde Ef é a eficiência do sistema (\%); VCA é o volume anual de chuva aproveitada; e VC é o volume anual de chuva.

Para se estabelecer um critério econômico para o sistema de água de chuva analisado, optou-se pela determinação do payback do investimento, o qual define o tempo necessário para que o capital investido seja recuperado pelo rendimento adquirido. Matematicamente definido pela razão entre o capital investido e o rendimento. Sendo, o capital investido CS; e o rendimento, a economia gerada com o sistema ES (Equação 6).

$$
\mathrm{Pb}=\mathrm{Cs} / \mathrm{Es}
$$


Onde $\mathrm{Pb}$ é o payback do investimento (anos); Cs é o Custo do sistema (R\$); e Es é a Economia gerada pelo sistema $(\mathrm{R} \$)$.

\section{RESULTADOS E DISCUSSÃO}

Foram realizadas simulações de volumes de reservatório com $78 \mathrm{~m}^{3}$ (Tabela 2), $150 \mathrm{~m}^{3}$ (Tabela 3) e $274 \mathrm{~m}^{3}$ (Tabela 4), buscando avaliar condições de atendimento parcial e total da demanda de água para fins não potáveis no bloco de aulas analisado da UFOPA.

Tabela 2 - Resultados mensais da aplicação do método de simulação para um reservatório com volume de $78 \mathrm{~m}^{3}$

\begin{tabular}{|c|c|c|c|c|c|c|c|c|c|}
\hline \multicolumn{10}{|c|}{ ANÁLISE DE SIMULAÇÃO DO RESERVATÓRIO } \\
\hline \multicolumn{2}{|c|}{ Coeficiente de runoff $(C R)=$} & 0,8 & & & & & & & \\
\hline \multicolumn{2}{|c|}{ Volume do reservatório $\left(\mathrm{m}^{\mathrm{s}}\right)=$} & 78 & & & & & & & \\
\hline Meses & $\begin{array}{l}\text { Chuva média } \\
\text { mensal } \\
(\mathrm{mm})\end{array}$ & $\begin{array}{c}\text { Demanda } \\
\text { mensal } \\
\left(\mathrm{m}^{3}\right) \\
\end{array}$ & $\begin{array}{c}\text { Área de } \\
\text { captaçăo } \\
\left(\mathrm{m}^{2}\right) \\
\end{array}$ & $\begin{array}{l}\text { Volume de } \\
\text { chuva mensal } \\
\left(\mathrm{m}^{3}\right)\end{array}$ & 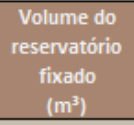 & $\begin{array}{c}\text { Volume do } \\
\text { reservatório no } \\
\text { tempo }(\mathrm{t}-1) \\
\left(\mathrm{m}^{3}\right)\end{array}$ & $\begin{array}{c}\text { Volume do } \\
\text { reservatório no } \\
\text { tempo }(\mathrm{t}) \\
\left(\mathrm{m}^{3}\right)\end{array}$ & $\begin{array}{l}\text { Overflow } \\
\left(\mathrm{m}^{3}\right) \\
\end{array}$ & $\begin{array}{c}\text { Suprimento de } \\
\text { água externo } \\
\left(\mathrm{m}^{3}\right)\end{array}$ \\
\hline Coluna 1 & Coluna 2 & Coluna 3 & Coluna 4 & Coluna 5 & Coluna 6 & Coluna 7 & Coluna 8 & Coluna 9 & Coluna 10 \\
\hline Janeiro & 257,3 & 132 & 1607 & 331 & 78 & 0 & 78 & 121 & 0 \\
\hline Fevereiro & 331,2 & 132 & 1607 & 426 & 78 & 78 & 78 & 294 & 0 \\
\hline Março & 436,5 & 132 & 1607 & 561 & 78 & 78 & 78 & 429 & 0 \\
\hline Abril & 397,5 & 132 & 1607 & 511 & 78 & 78 & 78 & 379 & 0 \\
\hline Maio & 326,8 & 132 & 1607 & 420 & 78 & 78 & 78 & 288 & 0 \\
\hline Junho & 150,2 & 132 & 1607 & 193 & 78 & 78 & 78 & 61 & 0 \\
\hline Julho & 101,6 & 132 & 1607 & 131 & 78 & 78 & 77 & 0 & 0 \\
\hline Agosto & 60,9 & 132 & 1607 & 78 & 78 & 77 & 23 & 0 & 0 \\
\hline Setembro & 31,2 & 132 & 1607 & 40 & 78 & 23 & -69 & 0 & 69 \\
\hline Outubro & 41,1 & 132 & 1607 & 53 & 78 & 0 & -79 & 0 & 79 \\
\hline Novembro & 65,5 & 132 & 1607 & 84 & 78 & 0 & -48 & 0 & 48 \\
\hline Dezembro & 131,5 & 132 & 1607 & 169 & 78 & 0 & 37 & 0 & 0 \\
\hline Total & 2331,3 & 1584 & & 2997 & & & & 1572 & 196 \\
\hline
\end{tabular}

Fonte: Autor (2020)

O sistema considerando um reservatório de $78 \mathrm{~m}^{3}$ pode suprir a edificação por um período de 9 meses (75\% do tempo) e um volume de utilização de $1425 \mathrm{~m}^{3}$. Na simulação também foi determinado o período de 3 meses (setembro, outubro e novembro), meses de estiagem na região. Nesse período, há necessidade de complementação de um volume de $196 \mathrm{~m}^{3}$ de fonte externa para atendimento da demanda, o valor extravasado não aproveitado foi de $1572 \mathrm{~m}^{3}$, o qual ocorre no primeiro semestre época chuvosa na região. 
Revista Tecnologia e Ambiente, v. 28, 2022, Criciúma, Santa Catarina/SC - ISSN Eletrônico 2358-9426 e ISSN Impresso 1413-8131

Tabela 3 - Resultados mensais da aplicação do método de simulação para um reservatório com volume de $150 \mathrm{~m}^{3}$

\begin{tabular}{|c|c|c|c|c|c|c|c|c|c|}
\hline \multicolumn{10}{|c|}{ ANÁLISE DE SIMULAÇÃO DO RESERVATÓRIO } \\
\hline \multicolumn{2}{|c|}{ Coeficiente de runoff $(C R)=$} & 0,8 & & & & & & & \\
\hline \multicolumn{2}{|c|}{ Volume do reservatório $\left(\mathrm{m}^{2}\right)=$} & 150 & & & & & & & \\
\hline Meses & $\begin{array}{c}\text { Chuva média } \\
\text { mensal } \\
(\mathrm{mm})\end{array}$ & $\begin{array}{c}\text { Demanda } \\
\text { mensal } \\
\left(\mathrm{m}^{3}\right) \\
\end{array}$ & $\begin{array}{l}\text { Área de } \\
\text { captação } \\
\left(\mathrm{m}^{2}\right) \\
\end{array}$ & $\begin{array}{c}\text { Volume de } \\
\text { chuva mensal } \\
\left(\mathrm{m}^{3}\right)\end{array}$ & $\begin{array}{c}\text { Volume do } \\
\text { reservatório } \\
\text { fixado } \\
\left(\mathrm{m}^{3}\right)\end{array}$ & $\begin{array}{c}\text { Volume do } \\
\text { reservatório no } \\
\text { tempo }(\mathrm{t}-1) \\
\left(\mathrm{m}^{3}\right) \\
\end{array}$ & $\begin{array}{c}\text { Volume do } \\
\text { reservatório no } \\
\text { tempo }(\mathrm{t}) \\
\left(\mathrm{m}^{3}\right) \\
\end{array}$ & $\begin{array}{l}\text { Overflow } \\
\left(\mathrm{m}^{3}\right)\end{array}$ & $\begin{array}{l}\text { Suprimento de } \\
\text { água externo } \\
\left(\mathrm{m}^{3}\right)\end{array}$ \\
\hline Coluna 1 & Coluna 2 & Coluna 3 & Coluna 4 & Coluna 5 & Coluna 6 & Coluna 7 & \begin{tabular}{l|l|} 
Coluna 8 \\
\end{tabular} & Coluna 9 & Coluna 10 \\
\hline Janeiro & 257,3 & 132 & 1607 & 331 & 150 & 0 & 150 & 49 & 0 \\
\hline Fevereiro & 331,2 & 132 & 1607 & 426 & 150 & 150 & 150 & 294 & 0 \\
\hline Março & 436,5 & 132 & 1607 & 561 & 150 & 150 & 150 & 429 & 0 \\
\hline Abril & 397,5 & 132 & 1607 & 511 & 150 & 150 & 150 & 379 & 0 \\
\hline Maio & 326,8 & 132 & 1607 & 420 & 150 & 150 & 150 & 288 & 0 \\
\hline Junho & 150,2 & 132 & 1607 & 193 & 150 & 150 & 150 & 61 & 0 \\
\hline Julho & 101,6 & 132 & 1607 & 131 & 150 & 150 & 149 & 0 & 0 \\
\hline Agosto & 60,9 & 132 & 1607 & 78 & 150 & 149 & 95 & 0 & 0 \\
\hline Setembro & 31,2 & 132 & 1607 & 40 & 150 & 95 & 3 & 0 & 0 \\
\hline Outubro & 41,1 & 132 & 1607 & 53 & 150 & 3 & -76 & 0 & 76 \\
\hline Novembro & 65,5 & 132 & 1607 & 84 & 150 & 0 & -48 & 0 & 48 \\
\hline Dezembro & 131,5 & 132 & 1607 & 169 & 150 & 0 & 37 & 0 & 0 \\
\hline Total & 2331,3 & 1584 & & 2997 & & & & 1500 & 124 \\
\hline
\end{tabular}

Fonte: Autor (2020)

Tabela 4 - Resultados mensais da aplicação do método de simulação para um reservatório com volume de $274 \mathrm{~m}^{3}$

\begin{tabular}{|c|c|c|c|c|c|c|c|c|c|}
\hline \multicolumn{10}{|c|}{ ANÁLISE DE SIMULAÇÃO DO RESERVATÓRIO } \\
\hline \multicolumn{2}{|c|}{ Coeficiente de runoff $(C R)=$} & 0,8 & & & & & & & \\
\hline \multicolumn{2}{|c|}{ Volume do reservatório $\left(\mathrm{m}^{\mathrm{s}}\right)=$} & 274 & & & & & & & \\
\hline Meses & $\begin{array}{c}\text { Chuva média } \\
\text { mensal } \\
(\mathrm{mm})\end{array}$ & $\frac{\begin{array}{c}\text { Demanda } \\
\text { mensal }\end{array}}{\left(\mathrm{m}^{3}\right)}$ & $\begin{array}{l}\text { Área de } \\
\text { captaçáo } \\
\left(\mathrm{m}^{2}\right)\end{array}$ & $\begin{array}{l}\text { Volume de } \\
\text { chuva mensal } \\
\qquad\left(\mathrm{m}^{3}\right)\end{array}$ & $\begin{array}{c}\text { Volume do } \\
\text { reservatório } \\
\text { fixado } \\
\left(\mathrm{m}^{3}\right)\end{array}$ & $\begin{array}{l}\text { Volume do } \\
\text { reservatório no } \\
\text { tempo }(\mathrm{t}-1) \\
\left(\mathrm{m}^{3}\right)\end{array}$ & $\begin{array}{l}\text { Volume do } \\
\text { reservatório no } \\
\text { tempo (t) } \\
\left(\mathrm{m}^{3}\right)\end{array}$ & $\begin{array}{l}\text { Overflow } \\
\left(\mathrm{m}^{3}\right)\end{array}$ & $\begin{array}{l}\text { Suprimento de } \\
\text { água externo } \\
\qquad\left(\mathrm{m}^{3}\right)\end{array}$ \\
\hline Coluna 1 & Coluna 2 & Coluna 3 & Coluna 4 & Coluna 5 & Coluna 6 & Coluna 7 & Coluna 8 & Coluna 9 & Coluna 10 \\
\hline Janeiro & 257,3 & 132 & 1607 & 331 & 274 & 0 & 274 & 0 & 0 \\
\hline Fevereiro & 331,2 & 132 & 1607 & 426 & 274 & 274 & 274 & 294 & 0 \\
\hline Março & 436,5 & 132 & 1607 & 561 & 274 & 274 & 274 & 429 & 0 \\
\hline Abril & 397,5 & 132 & 1607 & 511 & 274 & 274 & 274 & 379 & 0 \\
\hline Maio & 326,8 & 132 & 1607 & 420 & 274 & 274 & 274 & 288 & 0 \\
\hline Junho & 150,2 & 132 & 1607 & 193 & 274 & 274 & 274 & 61 & 0 \\
\hline Julho & 101,6 & 132 & 1607 & 131 & 274 & 274 & 273 & 0 & 0 \\
\hline Agosto & 60,9 & 132 & 1607 & 78 & 274 & 273 & 219 & 0 & 0 \\
\hline Setembro & 31,2 & 132 & 1607 & 40 & 274 & 219 & 127 & 0 & 0 \\
\hline Outubro & 41,1 & 132 & 1607 & 53 & 274 & 127 & 48 & 0 & 0 \\
\hline Novembro & 65,5 & 132 & 1607 & 84 & 274 & 48 & 0 & 0 & 0 \\
\hline Dezembro & 131,5 & 132 & 1607 & 169 & 274 & 0 & 37 & 0 & 0 \\
\hline Total & 2331,3 & 1584 & & 2997 & & & & 1451 & 0 \\
\hline
\end{tabular}

Fonte: Autor (2020)

As Tabelas 3 e 4 apresentam os resultados das simulações com volumes de $150 \mathrm{~m}^{3}$ e $274 \mathrm{~m}^{3}$, encontrando-se nos dois casos, respectivamente, atendimento de 10 e 12 meses, volumes captados de $1497 \mathrm{~m}^{3}$ e $1546 \mathrm{~m}^{3}$, volumes extravasados de $1500 \mathrm{~m}^{3}$ e $1451 \mathrm{~m}^{3}$, com necessidade de suprimento externo de $124 \mathrm{~m}^{3}$ e $0 \mathrm{~m}^{3}$. A análise quanto aos volumes simulados de $150 \mathrm{~m}^{3}$ indicam que somente o maior é capaz de atender aos 3 meses de estiagem, o que representaria um investimento muito alto. Assim, foi 
determinado o custo do sistema com o reservatório de $78 \mathrm{~m}^{3}$, tendo seu dimensionamento sido realizado através da escolha de 3 reservatórios de $26 \mathrm{~m}^{3}$, perfazendo o volume de $78 \mathrm{~m}^{3}$. Escolheu-se cisterna no modo enterrado, determinandose um reservatório superior de $10 \mathrm{~m}^{3}$ suspenso em estrutura de concreto armado.

\section{Análise financeira e eficiência do sistema}

A Tabela 5 apresenta o somatório dos custos dos insumos para a implantação das cisternas que atendem $75 \%$ da demanda estabelecida.

Tabela 5 - Valor do reservatório e seus componentes para o volume de $78 \mathrm{~m}^{3} \mathrm{e}$ configuração de 3 cisternas de $26 \mathrm{~m}^{3}$

\begin{tabular}{lrr}
\hline Insumo & Valor unitário $(\mathbf{R} \mathbf{\$})$ & Total $(\mathbf{R} \mathbf{\text { ) }}$ \\
\hline Filtro PEMD & $8.847,21$ & $8.847,21$ \\
Cisterna de $26 \mathrm{~m}^{3}$ & $7.049,63$ & $21.148,89$ \\
Mão de obra & $3.200,00$ & $3.200,00$ \\
Caixa d'água superior de $10 \mathrm{~m}^{3}$ & $5.205,18$ & $5.205,18$ \\
Escavação de $79 \mathrm{~m}^{3}$ & 8,69 & 686,51 \\
Reaterro de $15 \mathrm{~m}^{3}$ & 47,18 & 707,70 \\
Estrutura de $7,5 \mathrm{~m}^{3}$ de concreto & $2.487,43$ & $18.655,73$ \\
armado & & \\
Transporte da Cisterna & $4.000,00$ & $4.000,00$ \\
40 m de tubulações e conexões & 49,94 & $1.997,26$ \\
Conjunto moto-bomba de 2 CV & $1.937,26$ & $1.937,26$ \\
& Total & $66.386,08$ \\
& BDI de 25\% & $16.596,52$ \\
& Total Geral & $82.982,59$ \\
\hline
\end{tabular}

Fonte: Autor (2020)

Na Tabela 6 são apresentados os valores do custo da tarifa mensal de água (Figura 4), que seria paga pela UFOPA à concessionária de água, caso seu sistema de abastecimento não fosse próprio; e Payback (Equação 6), confiança do sistema (Equações 3 e 4) e eficiência do sistema (Equação 5). 
Tabela 6: Dados da análise de viabilidade do sistema de aproveitamento de água da chuva do prédio "Laranjão" na UFOPA

\begin{tabular}{lcc}
\hline Variável & & Valor \\
\cline { 1 - 1 } Custo da tarifa mensal (R\$) & & $2.159,01$ \\
Volume de água da chuva aproveitado $\left(\mathrm{m}^{3}\right)$ & & 1.425 \\
Payback (anos) & & 3,2 \\
Confiança do sistema (\%) & 75 \\
Eficiência do sistema (\%) & 47 \\
\hline
\end{tabular}

Para o cálculo do payback, foi dividido o valor do custo total do projeto (Tabela 5) pelo custo da tarifa anual, que é igual ao custo da tarifa mensal (Tabela 6) multiplicado por 12. O valor da confiança do sistema foi determinado com base nos 3 meses de estiagem para os quais o sistema não atendeu a demanda mensal (Tabela 2). Nesse caso, tem-se $\mathrm{Fr}=3 / 12=0,25$ (Equação 4) e $\mathrm{Rf}=(1-0,25)=0,25 \times 100=75 \%$ (Equação 3). Já o valor da eficiência do sistema foi determinado, considerando a economia anual de água (Tabela 6), ou seja, o volume de água anual aproveitada do sistema, dividida pelo volume anual de água, que poderia ser aproveitado pelo sistema (Tabela 2 - coluna 5). Nesse caso, tem-se Ef $=(1425 / 2997) \times 100=47 \%$.

Após simulações para determinação do reservatório do sistema, determinou-se um reservatório com volume de $274 \mathrm{~m}^{3}$ (Tabela 4). Esse valor atenderia a demanda integral de água para usos não potáveis da edificação. Entretanto, a dimensão desse reservatório impede sua implantação devido à falta de espaço às proximidades da edificação. Assim, optou-se pela simulação do reservatório com um volume de $78 \mathrm{~m}^{3}$, obtendo-se um custo de R \$ 82.982,59 para a implantação do sistema. Apesar do sistema de abastecimento da Universidade ser realizado por extração própria de poço profundo. Realizou-se a análise para ter uma confrontação com valor de mercado da Companhia de Saneamento, que para o consumo estipulado totalizou mensalmente uma tarifa de $\mathrm{R} \$ 2.159,01$. Foram considerados apenas os insumos necessários para a implantação do sistema não se considerando o custo de energia por ser um custo variável, que já é aplicado no sistema atual de água potável. E neste caso, com custo de energia maior, pois a água é bombeada para um reservatório posicionado em cota de altura mais elevada que $10 \mathrm{~m}$. Tomou-se como parâmetro de viabilidade econômica o payback simples, sendo igual a 3,2 anos, mostrando a viabilidade econômica do sistema de captação de água. Também, 
foram realizadas análises de confiança e eficiência do sistema com valores de, respectivamente, $75 \%$ e $47 \%$.

Quanto à análise dos outros dois parâmetros do tripé da sustentabilidade que são os aspectos social e ambiental, no que tange ao aspecto ambiental a redução de extrações de águas do aquífero permite maior capacidade de recomposição do mesmo, ajudando no aproveitamento nobre da água potável. No aspecto social, verifica-se que o volume aproveitado de água da chuva (Tabela 6) é considerável, podendo resultar em amortecimento de cheias, deixando de gerar danos e perdas devidos à impermeabilização e ineficiente drenagem das bacias hidrográficas urbanas. O amortecimento das chuvas também pode torna-se um aspecto econômico, caso haja implantação dos sistemas em larga escala, reduzindo as operações tapa buracos após os períodos de inverno. Com os sistemas, também, pode-se reduzir os investimentos em limpeza e drenagem urbana, viabilizando investimentos em outras demandas prioritárias. Também, a redução de volume de água escoando nas ruas, reduz os riscos de condução de patógenos causadores de doenças de veiculação hídrica, um exemplo é a leptospirose.

No aspecto de sustentabilidade social que são as atitudes tomadas individualmente e coletivamente de forma organizada com métodos e incentivos que proporcionem melhores qualidade de vida, dignidade, saúde redução de desperdícios etc. Implantações como as discutidas neste trabalho, por uma instituição que tem cunho social, educacional e promotor de estudos é de grande relevância para gerar mudanças de comportamento.

\section{CONCLUSÃO}

Assim, observando-se os aspectos econômico, social e ambiental, o sistema de captação de água da chuva do bloco de aulas "Laranjão" da UFOPA em Santarém/PA é viável. Pondera-se, ainda, que tais sistemas já são previstos pela instituição. Ficou demonstrado o potencial da edificação analisada quanto ao volume de captação de água de chuva para atender demandas não potáveis e a possibilidade, dependendo da análise caso a caso, de reaplicação da metodologia utilizada, de forma exclusiva, ou associada a outros sistemas como reuso em edificações recém-construídas ou em construção na instituição. Nesse contexto, o aproveitamento da água da chuva torna-se essencial, 
sendo uma redescoberta de prática milenar com características simples e que sofreu, principalmente, recentemente, em nosso país, o engajamento de autoridades na elaboração de leis e normas capazes de fomentar a implantação de sistemas de captação de água da chuva para fins potáveis e não potáveis.

\section{REFERÊNCIAS}

ABNT (Associação Brasileira de Normas Técnicas). Água de chuva - aproveitamento de cobertura em áreas urbanas para fins não potáveis - NBR 15527. Rio de Janeiro, 2019.

CARDOSO, R. N. C.; BLANCO, C. J. C; DUARTE, J. M. (2020). Technical and Financial Feasibility of Rainwater Harvesting Systems in Public Buildings in Amazon, Brazil. Journal of Cleaner Production, v. 260, p. 121054. https://doi.org/10.1080/15715124.2020.1776301.

COSANPA (Companhia de Saneamento do Pará). Tabelas de tarifas de água e esgoto. Disponível em: http://www.cosanpa.pa.gov.br/. Aceeso em 17 de janeiro de 2020.

DA CRUZ, W. M.; BLANCO, C. J. C. (2017). Aproveitamento de Água Pluvial para Fins Não Potáveis Residenciais em Rio Branco/AC. Perspectivas online: exatas e $\begin{array}{lllll}\text { engenharias, } & v \text {. } & 17, & \text { p. } & 12-24\end{array}$ https://ojs3.perspectivasonline.com.br/exatas_e_engenharia/article/view/1003/858.

IWA (International Water Association). IWA World Water Congress \& Exhibition 2018: Tokyo Report. Tokyo, 2018. Disponível em: 2018_IWA_WWCE-Tokyo_Report.pdf (iwanetwork.org). Acesso em 09 de junho de 2019.

LAMBERTUCCI, E. P.; RODRIGUES, C. A.; SUKADA, M. (2016). Soluções em drenagem urbana e controle de enchentes: métodos de gerenciamento e suas tecnologias. In: $1^{\mathbf{0}}$ Congresso Nacional de Iniciação Científica. CONIC/SEMESP. Disponível em: https://www.conic-semesp.org.br/anais/files/2016/trabalho1000021742.pdf. Aceeso em 17 de maio de 2021.

MAGALHÃES, G. B.; ZANELLA, M. E.; SALES, M. C. (2009). A ocorrência de chuvas e a incidência de leptospirose em Fortaleza-CE. Hygeia - Revista Brasileira de Geografia Médica e da Saúde, v. 5, n. 9, p. 77-87.

MUNDT, R.D.; NOGUEIRA, C.U.; NOGUEIRA, H.M.C.M.; FANTINEL, A.L.; CECONI, C.A.; LEAL, L. 2013. Aproveitamento da água da chuva para lavagem de máquinas. In: $\mathbf{2}^{\mathbf{0}}$ fórum internacional ecoinovar. Disponível em: http://ecoinovar.com.br/cd2013/arquivos/resumos/ECO158.pdf. Acesso em: 15 de setembro de 2021.

PEREIRA, G. S.; CAMILOTTO, S.; CONTO, S. M. (2019). Praticas Ambientais nos Estádios de Futebol da Copa do Mundo de 2014. Revista Eletrônica de Administração e Turismo - ReAT, v. 13, n.1, p. 1769-1786. HTTPS://DOI.ORG/10.15210/REAT.V13I1.14030. 
Revista Tecnologia e Ambiente, v. 28, 2022, Criciúma, Santa Catarina/SC - ISSN

Eletrônico 2358-9426 e ISSN Impresso 1413-8131

RUPP, R. F.; MUNARIM, U.; GHISI, E. (2011). Comparação de métodos para Dimensionamento de Reservatórios de Água Pluvial. Revista Ambiente Construido, v. 11, n.4, p. 47-64. https://doi.org/10.1590/S1678-86212011000400005. 